\title{
Evaluation of bioleaching column test of sulphide copper ore and copper concentrate using preconditioned surface
}

\author{
Alvaro Videla Leiva ${ }^{1, a}$, and Mario Vera Vélizand ${ }^{2}$ \\ ${ }^{1}$ Mining Engineering Department, Pontificia Universidad Catolica de Chile, Chile \\ ${ }^{2}$ Environmental Engineering Deparment, Pontificia Universidad Católica de Chile, Chile
}

\begin{abstract}
Bacterial activity can be related to the presence of $\mathrm{Fe}^{+3}$ in the solution, which drives copper oxidation during the secondary copper low grade sulfide ore leaching process. The present work evaluates improvements in kinetics of leaching when ferroxidans are preconditioned in an inert surface helping to build a biofilm which improves metabolism of the colony. The present work shows evaluation using laboratory columns to perform bioleaching during a 30 days period under three conditions: a base column with no inert surface, a column with loofa available for bacterial growth and conditioning, and finally a column with the loofa surface ground and distributed in the column among the particles.
\end{abstract}

a Corresponding author: avidela@ing.puc.cl 